\title{
MeSPL9 Attenuates Drought Resistance by Regulating JA Signaling and Protectant Metabolite Contents in Cassava
}

Shuxia Li ( $\sim$ lishuxia@itbb.org.cn )

Institute of Tropical Bioscience and Biotechnology https://orcid.org/0000-0001-7329-9259

\section{Zhihao Cheng}

Haikou Experimental Station

Zhibo Li

Institute of Tropical Bioscience and Biotechnology

Shiman Dong

Institute of Tropical Bioscience and Biotechnology

Xiaoling Yu

Institute of Tropical Bioscience and Biotechnology

Pingjuan Zhao

Institute of Tropical Bioscience and Biotechnology

Wenbin Liao

Institute of Tropical Bioscience and Biotechnology

Xiang Yu

Shanghai Jiao Tong University

Ming Peng

Institute of Tropical Bioscience and Biotechnology

\section{Research Article}

Keywords: Drought stress, MeSPL9, Jasmonic acid, Cassava, RNA-seq, Transcription factor

Posted Date: June 18th, 2021

DOl: https://doi.org/10.21203/rs.3.rs-609439/v1

License: (c) (i) This work is licensed under a Creative Commons Attribution 4.0 International License.

Read Full License

Version of Record: A version of this preprint was published at Theoretical and Applied Genetics on November 26th, 2021. See the published version at https://doi.org/10.1007/s00122-021-04000-z. 


\section{Abstract}

Drought stress severely impairs crop yield and is considered a primary threat to food security worldwide. Although the SQUAMOSA promoter binding protein-like $9(S P L 9)$ gene participates extensively in numerous developmental processes and in plant response to abiotic stimuli, its role and regulatory pathway in cassava (Manihot esculenta) response to the drought condition remain elusive. In the current study, we show that MeSPL 9 plays negative roles in drought stress resistance. MeSPL 9 expression was strongly repressed by drought treatment. Overexpression of a dominant-negative form of miR156resistant MeSPL9, rMeSPL9-SRDX, in which a 12-amino acid repressor sequence was fused to $r M e S P L 9$ at the $\mathrm{C}$ terminus, conferred drought tolerance without penalizing overall growth. $\mathrm{MeSPL9-SRDX-}$ overexpressing lines not only exhibited increased osmoprotectant metabolites including proline and anthocyanin, but also accumulated more endogenous jasmonic acid (JA) and soluble sugars. Transcriptomic and real-time PCR analysis suggested that differentially expressed genes were involved in sugar or JA biosynthesis, signaling, and metabolism in transgenic cassava under drought conditions. Exogenous application of JA further confirmed that JA conferred improved drought resistance and promoted stomatal closure in cassava leaves. Taken together, our findings suggest that MeSPL 9 affects drought resistance by modulating protectant metabolite levels and JA signaling, which have substantial implications for engineering drought tolerant crops.

\section{Key Message}

Analysis of drought-related genes in cassava shows the involvement of MeSPL9 in drought stress tolerance and overexpression of a dominant-negative form of this gene demonstrates its negative roles in drought stress resistance.

\section{Introduction}

Drought dramatically cuts crop yields, and becomes to a major threat to food security due to reduction in arable land area and reduced water availability worldwide (Ray et al. 2015). With the increase in the population of the earth, it is paramount to develop better-adapted crops in water-limiting environments to meet the requirement of world's food security and sustainability in future (Foley et al. 2011). Luckily, as sessile organisms, plants have evolved various defensive strategies to get through drought stress. Until recently, enormous progresses have been achieved to elucidate the responding mechanism of plants to drought stimulus and diverse genes enhancing drought tolerance have been introduced into crops, which is crucial for generating plants with increased drought resistance. It is also well documented that plants combat with drought stress by mobilizing transcriptional alteration of a number of genes, resulting in accumulation of key proteins, detoxification enzymes, phytohormones, or various metabolites, which contribute directly or indirectly to prevent plant cells from dehydration-induced damage (Fabregas et al. 2018; Krasensky and Jonak 2012; Seki et al. 2007). These studies reveal that drought tolerance is a complex trait concurrently mediated by a series of genes and pathways. 
The root crop cassava (Manihot esculenta) belongs to the Euphorbiaceae family and is the major food and bioenergy crop in the world. It is particularly important to the resource-poor farmers in arid regions, such as America, Asia, and Africa, owing to its relatively strong resistance to water- and nutrient-limited environments (E1-Sharkawy 2004; Okogbenin et al. 2013). However, harsh or persistent drought also significantly depresses the growth and development of cassava plants, thereby restricting their economic yield (E1-Sharkawy 2004). Our previous work reveals the involvement of the activation of jasmonic acid (JA) signaling pathway in drought-tolerant mechnisms of cassava ( $\mathrm{Li}$ et al. 2017a). The phytohormone $\mathrm{JA}$, as well as its metabolically active derivative JA-isoleucine (JA-lle), are critical signaling molecules, which orchestrate plant responses to biotic and abiotic stimuli (Riemann et al. 2015). Therefore, transgenic modulation of JA levels represents an attractive avenue to enhance the drought resistance of crops.

The essential components in JA biosynthesis and signaling have been widely investigated in plants. JA biosynthesis starts in the plastids and utilizes a-linolenic acids of chloroplast membranes as precursor. Oxygenation of a-linolenic acids by the enzyme 13-lipoxygenases (LOXs) to produce 13Shydroperoxyoctadecatrienoic acids (13-HPOTs), which is followed by the action of allene oxide synthases (AOSs) forms 12, 13S-epoxy-octadecatrienoic acids (12,13-EOTs). These are substrates for allene oxide cyclases (AOCs) that generate 12-oxophytodienoic acids (OPDAs). In the following, OPDAs are transported into peroxisomes where they are reduced by OPDA reductases (OPRs) and subsequently activated by CoA ester prior to undergoing three rounds of $\beta$-oxidation steps to form JA (Acosta and Farmer 2010; Wasternack and Hause 2013). Several reports suggest that endogenous JA and its precursor contents increased in plant cells under drought stress (Fu et al. 2018; Gupta et al. 2017; Savchenko et al. 2014). Exogenous application of JA and overexpression of the JA biosynthetic enzyme gene $L O X$ both could confer enhanced tolerance to drought conditions in plants; down-regulation of the $J A Z$ receptor also leads to drought-tolerant phenotypes (Fu et al. 2018; Xing et al. 2020). It has been proposed that JA improves drought tolerance by controlling stomatal aperture and increasing the antioxidant capacity of plants under osmotic stress (Riemann et al. 2015; Xing et al. 2020). Although the contributions of JA in plant response to environmental stresses have been suggested, the exact function of JA in cassava under drought stress condition remains controversial.

In cassava, previous studies have widely identified drought-responsive genes and characterized molecular mechanisms by using transcriptome profiling and genetic approaches. For example, one study documented that the contents of proline, malondialdehyde (MDA), and soluble sugars were all drastically increased during the PEG-simulated drought stress (Fu et al. 2016). Functions of drought-responsive genes such as dehydration-responsive element binding proteins (DREB) (An et al. 2017), heat shock protein (HSP) (Wei et al. 2020), and MYB (Ruan et al. 2017) have been verified and well characterized. Cassava plants have been conferred drought resistance using genetic approaches; however, this is accompanied reduced overall plant growth (An et al. 2017). Thus, identification of key genes that enhance stress tolerance without changing the architecture of plants is crucial for breeding crops with boosting growth in rain-fed environments. Here, we describe the characterization of one member of 
SQUAMOSA promoter binding protein-like (SPL) gene family, called MeSPL9, the downregulation of which can improve drought tolerance without affecting plant growth in cassava.

SPL proteins constitute a highly conserved plant-specific transcription factor family and play a key role in affecting plant development and optimizing plant response to stresses. A total of $16 S P L$ genes are identified in Arabidopsis, 10 of which are targets of miR156 (Guo et al. 2008). The role of miR156targeted $S P L s$ has been extensively explored by examining the phenotypes of plants expressing miR156resistant versions of these genes under the control of the constitutively expressed CaMV 35S promoter, or their own promoters (Wang et al. 2008). These overexpression phenotypes suggest that SPL proteins regulate a network of target genes and mediate various processes in plant development and physiology, encompassing the timing of vegetative phase change (Wu et al. 2009; Wu and Poethig 2006), anthocyanin biosynthesis (Gou et al. 2011; Wang et al. 2020), and root regeneration in tissue culture (Ye et al. 2020). On top of their functions in plant development, emerging evidence has proved that SPLs also serve as the main governing factors in response to environmental stresses. Studies have shown that miR156-mediated silencing of SPLs improved drought stress resilience by reducing water loss, while promoting leaf gas exchange and abscisic acid (ABA) sensitivity (Feyissa et al. 2019; Visentin et al. 2020). Transgenic Arabidopsis with increased miR156 expression silenced SPLs displayed enhanced tolerance under drought and salt conditions (Stief et al. 2014). In rice, miR156 overexpression inhibited the expression of miR156-targeted SPLs, resulting in enhanced resistance to $\mathrm{NaCl}$ and osmotic stresses (Cui et al. 2015).

In this study, we show that overexpression of a dominant-negative mutant MeSPL9-SRDX modulate multiple drought stress-related traits in cassava plants. Although the traits regulated by the miR156-SPL pathway are closely associated with plant development, we discovered that downregulation of MeSPL9 could enhance drought tolerance without penalizing overall plant growth. Our detailed transcriptomic and metabolite profiling showed that MeSPL 9-SRDX overexpression triggered JA accumulation and production of osmoprotectants (i.e., proline, soluble sugar, and anthocyanin), which are transcriptionally regulated by MeSPL 9 in cassava during drought condition. The findings would help in understanding the role of MeSPL 9 in plant response to drought stimulus and can be used as a tool in marker-assisted breeding to generate drought-resistant cassava and potentially other crops.

\section{Materials And Methods}

\section{Plant transformation and phenotype evaluation}

The full-length coding sequence (CDS) of cassava MeSPL 9 was cloned and mutated by PCR using specific primers harboring the miR156-targeted site (Table S3). The PCR fragment was confirmed by sequencing and subcloned into the pCAMBIA1301 binary vector under control of the CaMV 355 promoter, in which MeSPL 9 was fused with the EAR motif repressor domain (SRDX). The plasmid was introduced into Agrobacterium tumefaciens strain LBA4404, which was then transformed into cassava cultivar TMS60444 using friable embryogenic callus-mediated method (Zhang et al. 2000). The stem cuttings of 
wild-type (WT) and transgenic lines were cultured in MS plates and placed in a chamber $\left(26 \pm 2{ }^{\circ} \mathrm{C}, 16 / 8\right.$ $\mathrm{h}$ light/dark cycle) for three weeks. The seedlings were then planted in pots with high-quality soil substrates for treatments, or in Wenchang Plantation for Transgenic Crops, Hainan, China, for phenotype observation. The performance of at least ten plants per transgenic line and WT was recorded regularly till harvest.

\section{Southern blotting and quantitative real-time PCR (qRT-PCR)}

Total DNA was extracted from leaves of WT and transgenic lines by the CTAB method. Approximately 20 $\mu \mathrm{g}$ of isolated DNA were digested with EcoRI and separated on $0.8 \%(\mathrm{w} / \mathrm{v})$ agarose gels, followed by transferring to a positively charged nylon membrane (Roche, Mannheim, Germany). Then the membrane was hybridized with the DIG-labeled hygromycin phosphotransferase (HPT) probe. After hybridization, membrane washing and signal detection were performed by using the DIG-High Prime DNA Labeling and Detection Starter Kit II (Roche, Mannheim, Germany), following the manufacturer's protocols.

Total RNA isolation, cDNA synthesis, and qRT-PCR were conducted as previously described (Li et al. 2017b). Briefly, the differential gene expression between samples was assessed by the $\triangle \triangle C T$ method, with $M e A C T I N$ used as the reference gene. All samples were measured three times, and the experiments included three independent biological replicates. The primers are listed in Table S3.

\section{JA and drought treatments}

Three-week-old seedlings of WT and transgenic lines were planted in pots and grown for two weeks under a fully watered regime. For JA treatment, methyl JA (MeJA, Sigma-Aldrich) was dissolved in DMSO to 50 $\mathrm{mM}$ and diluted to a final concentration of $10 \mu \mathrm{M}$ with MS. WT seedlings were withhold water and were sprayed with mock or $10 \mu \mathrm{M}$ of MeJA once per week until the plants gradually dry out. For drought treatment, water was withdrawn for three weeks until the WT and transgenic lines exhibited obvious symptoms of damage. Each treatment contained at least six plants per line, and all treatments included three biological replicates.

\section{Proline quantification}

Four-week-old transgenic and WT lines grown in MS medium were divided into two groups (control and drought treatment); each independent sample consisted of at least five plants and all treatments included three biological replicates. After the plants were treated with 20\% PEG6000 for $6 \mathrm{~h}$, approximately $1 \mathrm{~g}$ of shoot tissues was collected for each sample and extracted with various reagents corresponding to different assays. Proline content was determined by the sulfosalicylic acid-acid ninhydrin method as previously described (Cheng et al. 2019).

\section{Determination of water loss rate and stomatal aperture}

To determine the water loss rate, fully expanded, healthy leaves of three-month-old WT and transgenic plants (6 leaves per repeat) were collected and immediately weighed (WF). Afterward, the leaves were 
placed in plastic trays at $26^{\circ} \mathrm{C}$ with $55 \%$ relative humidity. The weight (WD) of the leaves was measured in assigned intervals. The water loss rate (\%) was calculated as (WF-WD)/WF $\times 100 \%$. The experiment was conducted in triplicate.

Stomatal aperture was measured as previously described (Xing et al. 2020). Fully expanded mature leaves of transgenic and WT plants were incubated in stomatal opening solution for $2 \mathrm{~h}$ under light conditions. Afterward, the leaves were soaked in different solutions, including 20\% PEG 6000 and $10 \mu \mathrm{M}$ $\mathrm{JA}$, for $2 \mathrm{~h}$ prior to observation. The stomatal aperture was represented by averaging at least 60 stomata ratios (width/length).

\section{Quantification of endogenous hormones and soluble sugars}

Plant hormones, such as salicylic acid (SA), ABA, JA, and JA-lle, were determined in the shoots of fourweek-old WT and transgenic lines under control and drought conditions described above. Three replicates of samples were collected for each line, immediately frozen in liquid nitrogen, and stored at $-80^{\circ} \mathrm{C}$. A quantitative analysis of these endogenous hormones was conducted by high-performance liquid chromatography-mass spectrometry (HPLC-MS) as previously described (Fabregas et al. 2018). The standards ABA, SA, JA and JA-lle were purchased from Sigma-Aldrich (America).

Soluble sugar determination was undertaken according to the method described previously (Maloney et al. 2015). Briefly, shoot tissues (100 mg) were collected and homogenized in liquid nitrogen, dissolved in $6 \mathrm{ml}$ of methanol:chloroform:water $(12: 5: 3, \mathrm{v} / \mathrm{v})$, and incubated at $-20^{\circ} \mathrm{C}$ for $30 \mathrm{~min}$. Crude extracts were centrifuged at $6000 \mathrm{rpm}$ and $4{ }^{\circ} \mathrm{C}$ for $10 \mathrm{~min}$, and the supernatant was collected. The pellets were washed and re-suspended with methanol:chloroform:water (12:5:3, v/v). After centrifugation, the second supernatant was added to the first, $5 \mathrm{ml}$ of distilled water was added to the pooled supernatants and phases were partitioned. Aqueous phase that contained the soluble sugars was collected and dried. The soluble sugars were dissolved in distilled water and quantified by HPLC. The concentration of each component was measured with regression equations from calibration curves derived from external standards.

\section{RNA-seq analysis}

Young leaves and shoot tips from four-week-old WT and rMeSPL9-SRDX lines grown under control and drought (20\% PEG, 3h) conditions were harvested. Two biological replicates consisting of five independent plants for each sample were conducted. Total RNA isolation, library construction, and deep sequencing were performed on the NovaSeq ${ }^{\mathrm{TM}} 6000$ platform (Illumina) according to the manufacturer's protocols at the Guangzhou Genedenovo Biotechnology Co., Ltd. (Guangzhou, China). After removing the adapters and low-quality bases, clean reads were aligned to the cassava genome assembly with HISAT (Kim et al. 2015), followed by transcript construction using Stringtie (Pertea et al. 2015). The FPKM value was calculated for each unigene by RSEM (Li and Dewey 2011). The differentially expressed genes (DEGs) were identified by using DESeq2 (Love et al. 2014), with FDR $<0.05$. The RNA-seq data have been 
deposited to the National Center for Biotechnology Information (NCBI) under the accession number PRJNA693998.

\section{Statistical analyses}

All results were presented as mean \pm standard deviation (SD). Data were analyzed by one-way analysis of variance (ANOVA) and $t$-test in SPSS (IBM, New York, NY) and Microsoft Office Excel, considering *, $\mathrm{P}<$ $0.05, * *, P<0.01$, and $* * *, P<0.001$ as significance.

\section{Accession numbers}

MeSPL9 (Manes.09G032800), MeLOX2 (Manes.07G002400), MeLOX6 (Manes.10G149000), MeAOC (Manes.14G098200), MeAOS(Manes.18G031800), MeOPR2 (Manes.03G039700), MeOPR3 (Manes.18G120500), MeENO1 (Manes.01G023200), MeGPT2 (Manes.17G036800), MePK (Manes.17G052300), MeGAPC (Manes.12G015500), MeSPS (Manes.18G109400), MeINVA (Manes.02G035900).

\section{Results}

\section{Identification and expression pattern of MeSPL9 in cassava}

Bioinformatic analysis of the cassava genome identified 21 full-length $S P L s$, which is greater than the that (16) reported in Arabidopsis (Guo et al. 2008). All of the deduced MeSPL proteins, with lengths ranging from 148 to 1044 amino acids (aa), contained the conserved SBP domain (Table S1). A phylogenetic tree involving $21 \mathrm{MeSPLs}$ and 16 AtSPLs was constructed based on the neighbor-joining (NJ) method. The topology of the tree demonstrated that these SPLs were sparsely distributed in a majority of clades, and clustered into seven distinct groups, namely Group 1 to Group 7 (Fig. 1A). The MeSPLs were named MeSPL 1 to MeSPL 15 after the corresponding AtSPLs with highest sequence similarity. Remarkably, 10 AtSPLs are post-transcriptionally targeted by miRNA156 (Wu et al. 2009). Their closest homologs in cassava, including MeSPL3a/3b/4/5, MeSPL9/15, MeSPL2a/2b, and $S P L 6 a / 6 b / 6 c / 13 a / 13 b$, are all proved to be cleaved by mes-miR156 (Chen et al. 2015; Li et al. 2020).

To explore the potential function of $S P L$ genes, expression level changes of all $21 S P L s$ in cassava were analyzed based on our available RNA-Seq data ( $\mathrm{Li}$ et al. 2017a). The results showed that MeSPL9 was down-regulated especially by drought (Fig. 1B). The spatial expression pattern analysis revealed that MeSPL9 was preferentially expressed in leaves, stems and shoot apex, while lower expression levels could be detected in roots (Fig. 1C). The qRT-PCR was applied to further verify whether the MeSPL9 expression was related to drought stress. The results were overall in line with transcriptome data showing suppressed MeSPL 9 expression under drought conditions (Fig. 1D). The above results demonstrate that MeSPLs may play different roles in cassava under drought conditions, and lower MeSPL 9 expression is favored in cassava response to drought stress. 
We tried to generate MeSPL9 knockdown mutant plants using the chimeric repressor gene-silencing technology, in which mes-miR156 resistant MeSPL9 ( $M$ MeSPL9) was fused with a 12-aa SRDX under the control of constitutive 35S promoter (Fig. 2A) (Hiratsu et al. 2003). Previous studies demonstrated that this chimeric version can productively suppress the target genes (e.g., TCP genes) of various transcription factors (Guo et al. 2010; Koyama et al. 2007). Five independent transgenic cassava plant lines (named rMeSPL9-SRDX \#1 to \#5) harboring the MeSPL9-SRDX overexpressing cassette were generated by the use of Agrobacterium-mediated transformation of embryonic calli (Zhang et al. 2000). The basic transcriptional level of $r M e S P L 9-S R D X$ in each transgenic line was confirmed using qRT-PCR. \#2, \#3, and \#4 lines exhibited an exceptional up-regulation of $r M e S P L 9-S R D X$, approximately 60-fold higher relative to that of WT (Fig. 2B). The positive integration of the 35S::MeSPL 9-SRDXT-DNA was confirmed in transgenic lines using Southern blotting. Three lines were verified as positive insertion into the genome by ECoRI digestion (Fig. 2C). At the same time, we also tried to generate $M$ MeSPL9 overexpression transgenic plants using embryonic calli as receptors for transformation. However, the obtained calli failed to regenerate into plants, which is consistent with previous results that extremely high levels of SPL9 may lead to embryonic lethality (Wang et al. 2008). Therefore, \#2 and \#3, these two lines together with WT were used for subsequent studies. We carefully compared the growth performance of WT and transgenic plants. Generally, expression of rMeSPL9-SRDX had little effect on the development of cassava plant and were not obviously different from WT (Fig. 2D). However, we noticed hyperaccumulation of anthocyanin at the stem-petiole junction and petiole of $r M e S P L 9-S R D X$ plants (Fig. 2E). These results are in line with those obtained from the previous reports that $S P L 9$ participates in inhibiting anthocyanin biosynthesis in Arabidopsis (Gou et al. 2011; Wang et al. 2020).

\section{MeSPL9 negatively mediates drought tolerance at the seedling stage}

Cassava is established to be tolerant under drought conditions (Okogbenin et al. 2013), and it was of interest to assess whether rMeSPL9-SRDX overexpression could influence drought tolerance of transgenic plants. Therefore, five-week-old WT and rMeSPL 9-SRDX plants grown in pots were subjected to drought stress by water depletion. Prior to drought treatment, all plants displayed active growth status (Fig. 3A). After water depletion for three weeks, WT plants exhibited obvious dehydration symptoms including severe wilting and dehydrated leaves. In contrast, $r M e S P L 9-S R D X$ lines displayed more green leaves and relatively less severe dehydration. After rewatering, the rMeSPL9-SRDX plants exhibited increased drought tolerance with higher survival rates relative to WT (Fig. 3A, B). Detached leaves were subjected to dehydration to monitor the water loss rate. As can be seen in Fig. 3c, the WT leaves lost more water compared to the rMeSPL9-SRDX leaves during the entire experiment. Furthermore, proline content, a key indicator of plant drought adaptation (Dobra et al. 2011; Sperdouli and Moustakas 2012; Szabados and Savoure 2010), was markedly increased in the rMeSPL9-SRDX lines compared to WT (Fig. 3D). Collectively, the above results suggest that MeSPL9 negatively regulates drought stress adaptation in cassava.

\section{Identification of MeSPL9regulated genes and related pathways}


To elucidate the molecular mechanism underlying the enhanced drought tolerance of rMeSPL9-SRDX lines, we performed RNA-seq to investigate the transcriptomic changes between two genotypes (WT and rMeSPL9-SRDX lines) under two conditions (control and drought). To this end, the whole transcriptome profiles of $r M e S P L$ 9-SRDX and WT plants under control (designated \#2C, \#3C for rMeSPL 9-SRDX lines and WTC for wild-type) and drought treatment conditions (designated \#2D, \#3D for rMeSPL9-SRDX lines and WTD for wild-type) were compared. In WT, a total of 1,668 DEGs were detected under drought conditions. Among them, $76 \%$ of the genes $(1,263 / 1,668)$ were upregulated and $24 \%$ of the genes $(405 / 1,668)$ were downregulated (Fig. 4A, Table S2). In contrast, 1,940 and 2,385 drought-responsive DEGs were identified in $r M e S P L 9-S R D X$ line \#2 and \#3, respectively, among which approximately $71 \%$ of the genes were upregulated. These data suggested that there were larger shifts in gene expression under drought stress in $r M e S P L 9-S R D X$ lines. MeSPL9-regulated DEGs were determined by performing pairwise comparisons between rMeSPL9-SRDX lines and WT (\#2C vs. WTC, \#3C vs. WTC, \#2D vs. WTD and \#3D vs. WTD). As a result, 644 and 308 DEGs were detected in the \#2 and \#3 plants, respectively, under control conditions compared to WT. Similarly, 550 and 345 DEGs were found in these two lines, respectively, during drought treatment relative to WT (Fig. 4A, Table S2). We observed that the number of up-regulated DEGs was much greater than that of down-regulated DEGs in rMeSPL9-SRDX plants, suggesting that MeSPL9 may inhibit some of the drought-responsive genes.

Further investigation demonstrated that the expression of 15 and 30 genes were promoted and suppressed in rMeSPL9-SRDX lines in response to drought stress, respectively (Fig. 4B, C). Gene ontology (GO) enrichment analysis revealed that the DEGs belonging to 'response to abiotic/biotic stress' were overrepresented both in the control- and drought-treated rMeSPL9-SRDX plants (Fig. 4D, E). To further elucidate the roles of the MeSPL9-regulated DEGs, KEGG enrichment analysis was performed.

Anthocyanin biosynthesis and proline metabolism were significantly up-regulated in rMeSPL9-SRDX lines under control condition. However, the majority of genes related to monoterpenoid biosynthesis were strongly up-regulated after drought treatment. It is noteworthy that genes involved in alpha-linolenic acid metabolism were notably overrepresented under both control and drought conditions (Fig. 4F, G). The biosynthetic process of hormone JA using alpha-linolenic acid as substrates in chloroplast has been extensively studied (Han 2017; Wasternack and Hause 2013). The above results indicated that the DEGs involved in plant metabolism and JA signaling pathways may play key roles during drought stress.

\section{JA participates in regulation of stomatal closure in rMeSPL9-SRDX plants}

Our KEGG analysis revealed that MeSPL 9 may repress JA biosynthesis and signaling in cassava plants. Indeed, previous reports have demonstrated that $S P L s$ can attenuate JA responses by stabilizing JAZ proteins (Mao et al. 2017), the repressor of JA signaling pathway. As we know, JA function as an important regulator in plant development and response to abiotic stimuli, including drought, as well as biotic stimuli (Ahmad et al. 2016; Wasternack and Strnad 2016). In this study, we further found that a number of drought-induced DEGs involved in JA biosynthesis, catabolism, and signaling processes were significantly up-regulated in IMeSPL 9-SRDX plants under both control and drought conditions compared with WT according to the transcriptional data (Fig. 5A, B). To validate the results obtained from the 
transcriptomic analysis, we analyzed the expression of various JA biosynthesis-related genes by qRTPCR. The expression of MeLOXs, MeAOS, MeAOC, and MeOPRs in rMeSPL 9-SRDX lines was continuously induced before and after drought treatment, which is mostly consistent with the RNA-seq results (Fig. S1A-F). These findings prompt us to evaluate the endogenous JA level in transgenic cassava plants. As shown in Fig. 5c, no significant differences in SA and ABA contents were observed between transgenic and WT plants, while JA content was markedly induced in $r M e S P L$ 9-SRDX lines compared to WT, indicating that MeSPL9 may negatively regulate the synthesis of endogenous JA.

Previous reports have shown that JA promotes drought resistance of plants by moderating stomatal closure (Xing et al. 2020). We therefore postulate that JA accumulation may confer enhanced drought resistance in rMeSPL9-SRDX plants. To test this postulation, exogenous JA was applied to wild-type cassava seedlings before drought treatment. As a result, seedlings sprayed exogenous MeJA exhibited higher survival rate after drought treatment compared with the control (Fig. 5D, F), indicating that JA was able to increase drought resistance in cassava. Meanwhile we examined the stomatal aperture of both WT and rMeSPL 9-SRDX leaves treated with PEG and JA, respectively. As shown in Fig. 5e, smaller stomatal aperture was observed in rMeSPL 9-SRDX leaves compared with WT under normal conditions, which corresponded to the lower water loss rates as mentioned above. Stomatal aperture assays also revealed that both exogenous JA and PEG treatment can inhibit stomatal opening in WT and transgenic leaves, whereas $r M e S P L$ 9-SRDX conferred a relative lower stomatal closing capacity than WT and showed hyposensitivity to exogenous JA (Fig. 5E, G). Taken together, these results suggested that the elevated JA levels, especially in rMeSPL 9-SRDX plants, are responsible for stomatal closure in order to prevent water loss, thereby leading to drought resistance.

\section{rMeSPL9-SRDX plants contain higher levels of soluble sugars}

To gain additional insight into the mechanism underlying drought resistance conferred by rMeSPL9-SRDX overexpression, we analyzed soluble sugar levels in PEG treated leaves. By comparison, the sugar contents in $r M e S P L 9-S R D X$ leaves, such as glucose, fructose and galactose were significantly higher to the WT under control condition, and were elevated even further as a result of drought treatment (Fig. 6AC). However, no difference in sucrose levels was detected between WT and rMeSPL9-SRDX plants (Fig. $6 \mathrm{D})$. This result suggested that accumulation of soluble sugar contents could serve as another indicator for evaluating the drought response in $M$ MeSPL9-SRDX leaves. When combined with the transcriptome data presented above, the genes related to carbon metabolism and plant glycolysis pathway appear to be positively associated with drought response. Subsequently, we observed the expression of genes encoding enolase 1 (ENO1), glucose 6-phosphate/phosphate translocator 2 (GPT2), pyruvate kinase (PK), and vacuolar invertase (INVA) in the starch and sucrose metabolism pathways was remarkably upregulated in rMeSPL 9-SRDX plants (Fig. 6E). Glyceraldehyde-3-phosphate dehydrogenases (GAPCS) are ubiquitous proteins that function in the glycolytic pathway and are involved in stress response (Guo et al. 2012; Li et al. 2019b). In rMeSPL9-SRDX plants, the expression level of MeGAPC was also significantly increased. Moreover, we also discovered down-regulated genes by overexpressing rMeSPL9-SRDX, such as sucrose-phosphate synthase (SPS), which serves as one of the rate-limiting steps in sucrose synthesis 
in plants (Fig. 6E). Additionally, the expression of MeENO1, MeGPT2, MePK, and MeGAPC was notably induced under drought stress. However, no particular difference in the expression of MeINVA and MeSPS was observed between control and drought conditions.

\section{Discussion}

Although cassava plants is often classified as drought tolerant due to that they are able to grow through the dry seasons in tropical areas, persistent drought stress seriously restricts its growth and production (E1-Sharkawy 2004). Using high-throughput RNA-seq, numerous drought-responsive genes and related regulatory networks have been identified in cassava (Fu et al. 2016; Li et al. 2017a; Utsumi et al. 2012). It is currently proposed that the strategy in cassava response to water stress is 'avoiding drought' through enhancing root growth and encouraging leaf abscission to reduce water transpiration (Liao et al. 2016; Okogbenin et al. 2013). In the present study, we reported that MeSPL 9 acted as a negative regulator in cassava response to drought stress. Although several studies have confirmed in various species the participation of SPLs in plant response to drought stress (Cui et al. 2015; Feyissa et al. 2019; Visentin et al. 2020), the underlying mechanisms and downstream targets remain largely unclear. Here we show that the transgenic plants overexpressing rMeSPL9-SRDX, a dominant-negative chimeric gene, exhibited a representative drought resistant phenotype. It is suggested that this phenomenon was achieved by the accumulation of protectant metabolites, such as phytohormone JA, anthocyanidin, proline, and soluble sugars, through transcriptional regulation of metabolic pathways.

On top of participating in plant response to abiotic stimuli, miR156-targeted SPLs are largely conserved among various plant species and proposed to play a prominent role in the regulation of plant growth and development, including phase change, root regeneration, and wax synthesis (Li et al. 2019a; Wang et al. 2008; Wu et al. 2009; Ye et al. 2020). Previously, miR156 overexpression enhanced the tolerance to drought stress in plants, but also impaired the overall growth of transgenic plants (Cui et al. 2015; Feyissa et al. 2019). In Arabidopsis, single-knockout mutants of miR156-targeted SPL genes exhibited no significant morphological phenotype changes (Wang et al. 2008). Similarly, in cassava, constitutive expression of $r M e S P L 9-S R D X$ can promote drought resistance without altering the growth features of transgenic plants. Thus, characterization of MeSPL9-related genes and pathways in cassava plants are benefit for generating drought-tolerant cultivators.

Under control and drought conditions, rMeSPL9-SRDX lines showed enrichments of proline and anthocyanins. These two metabolites, as important antioxidants, contribute significantly to osmotic homeostasis and protection of macromolecules in plants suffering from abiotic stresses (Sperdouli and Moustakas 2012; Szabados and Savoure 2010). Increased anthocyanin or proline production has been demonstrated to significantly enhance resistance to abiotic stimuli in various plant species (Castellarin et al. 2007; Lotkowska et al. 2015; Naing et al. 2017). Indeed, a previous study revealed that SPL9 may negatively regulate anthocyanin production via directly inhibiting the expression of anthocyanin biosynthesis-related genes by destabilization of a MYB-bHLH-WD40 transcriptional activation complex 
in Arabidopsis (Gou et al. 2011). Therefore, the enhanced accumulation of proline and anthocyanins strongly correlated with the drought stress tolerance of $r M e S P L 9-S R D X$ plants.

JA is the key phytohormone that positively regulates biotic and abiotic stress adaptation. A number of reports have demonstrated that JA accumulation can happen immediately when plants are subjected to abiotic stresses (Riemann et al. 2015). Overexpression of JA biosynthesis genes or exogenous application with JA could significantly enhance water stress tolerance by promoting the antioxidant enzyme activation or regulating the plant growth and tissue water status (Ozturk et al. 2015; Riemann et al. 2015; Wasternack and Strnad 2016). For instance, overexpression of the JA biosynthesis gene LOX and AOS can confer drought stress resistance (De Domenico et al. 2012; Xing et al. 2020). In this study, the rMeSPL9-SRDX lines exhibited increased JA content due to higher expression level of key JA biosynthesis-related genes, including MeLOXs, MeAOS and MeAOCs, compared with the WT plants. It has also been reported that JA could decrease transpiration loss by the regulation of stomatal closure (Daszkowska-Golec and Szarejko 2013; Suhita et al. 2004). In cassava, we provided evidence that JA treatment could magnify stomatal closure. Comparing with WT, rMeSPL9-SRDX lines exhibited lower water loss rates and smaller stomata aperture. Taken together, these results indicate that rMeSPL9related stress tolerance is partially JA-dependent in cassava.

Soluble sugars, act not only as an energy source, but also as osmolytes to maintain normal transpiration and leaf-water content under drought conditions (Wei et al. 2019). In this regard, the improvement of drought resistance by overexpressing rMeSPL9-SRDX has further encouraged us to evaluate the changes in concentration of soluble sugars in the leaves. Consistent with the previous studies (Pinheiro et al. 2011; Wei et al. 2019; Wingler and Roitsch 2008), while no differences in sucrose content were observed in rMeSPL9-SRDX lines, the contents of glucose, fructose, and galactose significantly increased. In our study, expression of starch-sucrose metabolism-related genes, such as MeENO1, MeGPT2, MePK and MeGAPC were higher in $r M e S P L 9-S R D X$ than in WT plants during drought conditions. Evidence for GAPC enzymes in plant response to abiotic stresses has come from a number of independent studies. They have been demonstrated to transduce the ROS hydrogen peroxide $\left(\mathrm{H}_{2} \mathrm{O}_{2}\right)$ signal in Arabidopsis by

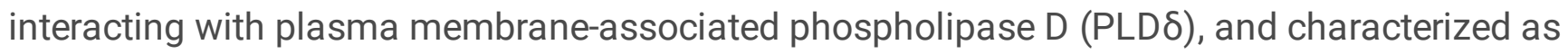
cytoplasmic proteins that have important role in ABA signaling pathway. The loss of GAPCs exhibited decreased stomatal sensitivity to $A B A$ and rendered plants less responsive to water deficits than the wild type (Guo et al. 2012). Therefore, when the MeGAPC activity is raised in transgenic lines, the in planta ROS signaling may be altered, resulting in improved drought tolerance. In plants, ENO1 catalyzes the glycolytic conversion of 2-phosphoglycerate (2-PGA) to phosphoenolpyruvate (PEP), which is an intermediate of glycolysis and delivers ATP and pyruvate with the presence of cytosolic PK (Prabhakar et al. 2010). GPT2 has previously been demonstrated to participate in regulating the metabolite distribution between compartments and to play a crucial role in interpreting environmental signals (Dyson et al. 2015). The enhanced MeENO1, MePK and MeGPT2 activities in rMeSPL9-SRDX plants would produce more soluble carbohydrates, which could be beneficial since they serve as osmoticum under drought stress. Meanwhile, due to the altered activities of MeSPS and MeINVA manifest in the 
synthesis/hydrolysis of sucrose, the overall levels of glucose and fructose were increased, but without an alteration in sucrose content. INVA has been demonstrated to facilitate water influx and maintain desired osmotic homeostasis by hydrolyzing sucrose into hexoses under abiotic stress conditions (Dahro et al. 2016). Due to this mechanism, it appears that higher levels of glucose and fructose in rMeSPL9-SRDX plants, might contribute to the enhanced drought tolerance.

Taken together, in this study, we isolated and characterized the novel function of MeSPL 9 in the regulation of drought adaptation of cassava plants. Our studies demonstrate that MeSPL9 negatively regulates drought tolerance through controlling anthocyanin biosynthesis, JA, proline and soluble sugars accumulation. Further research will be focused on the identification of the molecular network of MeSPL9 in the regulation of stress adaptation.

\section{Declarations}

\section{Acknowledgments}

This work was supported by the Hainan Provincial Natural Science Foundation of China (320MS097), the National Key Research and Development Program of China (2019YFD1001105, 2019YFD1000500, 2018YFD1000500), and the Central Public-interest Scientific Institution Basal Research Fund for Chinese Academy of Tropical Agricultural Sciences (1630052021026).

\section{Author's contribution}

M.P. and X.Y. designed the experiments; S.L., Z.L., S.D., X.Y., P.Z., Z.C., and W.L. conducted the experiments; S.L., X.Y., and Z.C. wrote the manuscript. We would like to thank TopEdit (www.topeditsci.com) for its linguistic assistance during the preparation of this manuscript.

Conflict of interest The authors declare that there is no conflict of interest.

\section{References}

1. Acosta IF, Farmer EE (2010) Jasmonates. Arabidopsis Book 8:e0129

2. Ahmad P, Rasool S, Gul A, Sheikh SA, Akram NA, Ashraf M, Kazi AM, Gucel S (2016) Jasmonates: Multifunctional Roles in Stress Tolerance. Frontiers in plant science 7:813

3. An D, Ma Q, Wang H, Yang J, Zhou W, Zhang P (2017) Cassava C-repeat binding factor 1 gene responds to low temperature and enhances cold tolerance when overexpressed in Arabidopsis and cassava. Plant molecular biology 94:109-124

4. Castellarin SD, Pfeiffer A, Sivilotti P, Degan M, Peterlunger E, G DIG (2007) Transcriptional regulation of anthocyanin biosynthesis in ripening fruits of grapevine under seasonal water deficit. Plant, cell \& environment 30:1381-1399 
5. Chen X, Xia J, Xia Z, Zhang H, Zeng C, Lu C, Zhang W, Wang W (2015) Potential functions of microRNAs in starch metabolism and development revealed by miRNA transcriptome profiling of cassava cultivars and their wild progenitor. BMC plant biology 15:33

6. Cheng Z, Lei N, Li S, Liao W, Shen J, Peng M (2019) The regulatory effects of MeTCP4 on cold stress tolerance in Arabidopsis thaliana: A transcriptome analysis. Plant physiology and biochemistry : PPB 138:9-16

7. Cui LG, Shan JX, Shi M, Gao JP, Lin HX (2015) The miR156-SPL9-DFR pathway coordinates the relationship between development and abiotic stress tolerance in plants. The Plant journal : for cell and molecular biology 80:1108-1117

8. Dahro B, Wang F, Peng T, Liu JH (2016) PtrA/NINV, an alkaline/neutral invertase gene of Poncirus trifoliata, confers enhanced tolerance to multiple abiotic stresses by modulating ROS levels and maintaining photosynthetic efficiency. BMC plant biology 16:76

9. Daszkowska-Golec A, Szarejko I (2013) Open or close the gate - stomata action under the control of phytohormones in drought stress conditions. Frontiers in plant science 4:138

10. De Domenico S, Bonsegna S, Horres R, Pastor V, Taurino M, Poltronieri P, Imtiaz M, Kahl G, Flors V, Winter P, Santino A (2012) Transcriptomic analysis of oxylipin biosynthesis genes and chemical profiling reveal an early induction of jasmonates in chickpea roots under drought stress. Plant physiology and biochemistry : PPB 61:115-122

11. Dobra J, Vankova R, Havlova M, Burman AJ, Libus J, Storchova H (2011) Tobacco leaves and roots differ in the expression of proline metabolism-related genes in the course of drought stress and subsequent recovery. Journal of plant physiology 168:1588-1597

12. Dyson BC, Allwood JW, Feil R, Xu Y, Miller M, Bowsher CG, Goodacre R, Lunn JE, Johnson GN (2015) Acclimation of metabolism to light in Arabidopsis thaliana: the glucose 6-phosphate/phosphate translocator GPT2 directs metabolic acclimation. Plant, cell \& environment 38:1404-1417

13. E1-Sharkawy MA (2004) Cassava biology and physiology. Plant molecular biology 56:481-501

14. Fabregas N, Lozano-Elena F, Blasco-Escamez D, Tohge T, Martinez-Andujar C, Albacete A, Osorio S, Bustamante M, Riechmann JL, Nomura T, Yokota T, Conesa A, Alfocea FP, Fernie AR, Cano-Delgado Al (2018) Overexpression of the vascular brassinosteroid receptor BRL3 confers drought resistance without penalizing plant growth. Nature communications 9:4680

15. Feyissa BA, Arshad M, Gruber MY, Kohalmi SE, Hannoufa A (2019) The interplay between miR156/SPL13 and DFR/WD40-1 regulate drought tolerance in alfalfa. BMC plant biology 19:434

16. Foley JA, Ramankutty N, Brauman KA, Cassidy ES, Gerber JS, Johnston M, Mueller ND, O'Connell C, Ray DK, West PC, Balzer C, Bennett EM, Carpenter SR, Hill J, Monfreda C, Polasky S, Rockstrom J, Sheehan J, Siebert S, Tilman D, Zaks DP (2011) Solutions for a cultivated planet. Nature 478:337342

17. Fu J, Wu H, Ma S, Xiang D, Liu R, Xiong L (2018) OsJAZ1 Attenuates Drought Resistance by Regulating JA and ABA Signaling in Rice. Frontiers in plant science 8:2108 
18. Fu L, Ding Z, Han B, Hu W, Li Y, Zhang J (2016) Physiological Investigation and Transcriptome Analysis of Polyethylene Glycol (PEG)-Induced Dehydration Stress in Cassava. International journal of molecular sciences 17:283

19. Gou JY, Felippes FF, Liu CJ, Weigel D, Wang JW (2011) Negative regulation of anthocyanin biosynthesis in Arabidopsis by a miR156-targeted SPL transcription factor. The Plant cell 23:15121522

20. Guo AY, Zhu QH, Gu X, Ge S, Yang J, Luo J (2008) Genome-wide identification and evolutionary analysis of the plant specific SBP-box transcription factor family. Gene 418:1-8

21. Guo L, Devaiah SP, Narasimhan R, Pan X, Zhang Y, Zhang W, Wang X (2012) Cytosolic glyceraldehyde-3-phosphate dehydrogenases interact with phospholipase Ddelta to transduce hydrogen peroxide signals in the Arabidopsis response to stress. The Plant cell 24:2200-2212

22. Guo Z, Fujioka S, Blancaflor EB, Miao S, Gou X, Li J (2010) TCP1 modulates brassinosteroid biosynthesis by regulating the expression of the key biosynthetic gene DWARF4 in Arabidopsis thaliana. The Plant cell 22:1161-1173

23. Gupta A, Hisano H, Hojo Y, Matsuura T, Ikeda Y, Mori IC, Senthil-Kumar M (2017) Global profiling of phytohormone dynamics during combined drought and pathogen stress in Arabidopsis thaliana reveals $A B A$ and JA as major regulators. Scientific reports 7:4017

24. Han GZ (2017) Evolution of jasmonate biosynthesis and signaling mechanisms. Journal of experimental botany 68:1323-1331

25. Hiratsu K, Matsui K, Koyama T, Ohme-Takagi M (2003) Dominant repression of target genes by chimeric repressors that include the EAR motif, a repression domain, in Arabidopsis. The Plant journal : for cell and molecular biology 34:733-739

26. Kim D, Langmead B, Salzberg SL (2015) HISAT: a fast spliced aligner with low memory requirements. Nature methods 12:357-360

27. Koyama T, Furutani M, Tasaka M, Ohme-Takagi M (2007) TCP transcription factors control the morphology of shoot lateral organs via negative regulation of the expression of boundary-specific genes in Arabidopsis. The Plant cell 19:473-484

28. Krasensky J, Jonak C (2012) Drought, salt, and temperature stress-induced metabolic rearrangements and regulatory networks. Journal of experimental botany 63:1593-1608

29. Li B, Dewey CN (2011) RSEM: accurate transcript quantification from RNA-Seq data with or without a reference genome. BMC bioinformatics 12:323

30. Li RJ, Li LM, Liu XL, Kim JC, Jenks MA, Lu S (2019a) Diurnal Regulation of Plant Epidermal Wax Synthesis through Antagonistic Roles of the Transcription Factors SPL9 and DEWAX. The Plant cell 31:2711-2733

31. Li S, Cheng z, Peng M (2020) Genome-wide identification of miRNAs targets involved in cold response in cassava. Plant Omics Journal 13:57-64

32. Li S, Yu X, Cheng Z, Yu X, Ruan M, Li W, Peng M (2017a) Global Gene Expression Analysis Reveals Crosstalk between Response Mechanisms to Cold and Drought Stresses in Cassava Seedlings. 
Frontiers in plant science 8:1259

33. Li S, Yu X, Lei N, Cheng Z, Zhao P, He Y, Wang W, Peng M (2017b) Genome-wide identification and functional prediction of cold and/or drought-responsive IncRNAs in cassava. Scientific reports 7:45981

34. Li X, Wei W, Li F, Zhang L, Deng X, Liu Y, Yang S (2019b) The Plastidial Glyceraldehyde-3-Phosphate Dehydrogenase Is Critical for Abiotic Stress Response in Wheat. International journal of molecular sciences 20

35. Liao W, Wang G, Li Y, Wang B, Zhang P, Peng M (2016) Reactive oxygen species regulate leaf pulvinus abscission zone cell separation in response to water-deficit stress in cassava. Scientific reports 6:21542

36. Lotkowska ME, Tohge T, Fernie AR, Xue GP, Balazadeh S, Mueller-Roeber B (2015) The Arabidopsis Transcription Factor MYB112 Promotes Anthocyanin Formation during Salinity and under High Light Stress. Plant physiology 169:1862-1880

37. Love MI, Huber W, Anders S (2014) Moderated estimation of fold change and dispersion for RNA-seq data with DESeq2. Genome biology 15:550

38. Maloney VJ, Park JY, Unda F, Mansfield SD (2015) Sucrose phosphate synthase and sucrose phosphate phosphatase interact in planta and promote plant growth and biomass accumulation. Journal of experimental botany 66:4383-4394

39. Mao YB, Liu YQ, Chen DY, Chen FY, Fang X, Hong GJ, Wang LJ, Wang JW, Chen XY (2017) Jasmonate response decay and defense metabolite accumulation contributes to age-regulated dynamics of plant insect resistance. Nature communications 8:13925

40. Naing AH, Park KI, Ai TN, Chung MY, Han JS, Kang YW, Lim KB, Kim CK (2017) Overexpression of snapdragon Delila (Del) gene in tobacco enhances anthocyanin accumulation and abiotic stress tolerance. BMC plant biology 17:65

41. Okogbenin E, Setter TL, Ferguson M, Mutegi R, Ceballos H, Olasanmi B, Fregene M (2013) Phenotypic approaches to drought in cassava: review. Frontiers in physiology 4:93

42. Ozturk B, Yildiz K, Kucuker E (2015) Effect of pre-harvest methyl jasmonate treatments on ethylene production, water-soluble phenolic compounds and fruit quality of Japanese plums. J Sci Food Agric 95:583-591

43. Pertea M, Pertea GM, Antonescu CM, Chang TC, Mendell JT, Salzberg SL (2015) StringTie enables improved reconstruction of a transcriptome from RNA-seq reads. Nature biotechnology 33:290-295

44. Pinheiro C, Antonio C, Ortuno MF, Dobrev PI, Hartung W, Thomas-Oates J, Ricardo CP, Vankova R, Chaves MM, Wilson JC (2011) Initial water deficit effects on Lupinus albus photosynthetic performance, carbon metabolism, and hormonal balance: metabolic reorganization prior to early stress responses. Journal of experimental botany 62:4965-4974

45. Prabhakar V, Lottgert T, Geimer S, Dormann P, Kruger S, Vijayakumar V, Schreiber L, Gobel C, Feussner K, Feussner I, Marin K, Staehr P, Bell K, Flugge UI, Hausler RE (2010) Phosphoenolpyruvate provision 
to plastids is essential for gametophyte and sporophyte development in Arabidopsis thaliana. The Plant cell 22:2594-2617

46. Ray DK, Gerber JS, MacDonald GK, West PC (2015) Climate variation explains a third of global crop yield variability. Nature communications 6:5989

47. Riemann M, Dhakarey R, Hazman M, Miro B, Kohli A, Nick P (2015) Exploring Jasmonates in the Hormonal Network of Drought and Salinity Responses. Frontiers in plant science 6:1077

48. Ruan MB, Guo X, Wang B, Yang YL, Li WQ, Yu XL, Zhang P, Peng M (2017) Genome-wide characterization and expression analysis enables identification of abiotic stress-responsive MYB transcription factors in cassava (Manihot esculenta). Journal of experimental botany 68:3657-3672

49. Savchenko T, Kolla VA, Wang CQ, Nasafi Z, Hicks DR, Phadungchob B, Chehab WE, Brandizzi F, Froehlich J, Dehesh K (2014) Functional convergence of oxylipin and abscisic acid pathways controls stomatal closure in response to drought. Plant physiology 164:1151-1160

50. Seki M, Umezawa T, Urano K, Shinozaki K (2007) Regulatory metabolic networks in drought stress responses. Current opinion in plant biology 10:296-302

51. Sperdouli I, Moustakas M (2012) Interaction of proline, sugars, and anthocyanins during photosynthetic acclimation of Arabidopsis thaliana to drought stress. Journal of plant physiology 169:577-585

52. Stief A, Altmann S, Hoffmann K, Pant BD, Scheible WR, Baurle I (2014) Arabidopsis miR156 Regulates Tolerance to Recurring Environmental Stress through SPL Transcription Factors. The Plant cell 26:1792-1807

53. Suhita D, Raghavendra AS, Kwak JM, Vavasseur A (2004) Cytoplasmic alkalization precedes reactive oxygen species production during methyl jasmonate- and abscisic acid-induced stomatal closure. Plant physiology 134:1536-1545

54. Szabados L, Savoure A (2010) Proline: a multifunctional amino acid. Trends in plant science 15:8997

55. Utsumi Y, Tanaka M, Morosawa T, Kurotani A, Yoshida T, Mochida K, Matsui A, Umemura Y, Ishitani M, Shinozaki K, Sakurai T, Seki M (2012) Transcriptome analysis using a high-density oligomicroarray under drought stress in various genotypes of cassava: an important tropical crop. DNA research : an international journal for rapid publication of reports on genes and genomes 19:335-345

56. Visentin I, Pagliarani C, Deva E, Caracci A, Tureckova V, Novak O, Lovisolo C, Schubert A, Cardinale F (2020) A novel strigolactone-miR156 module controls stomatal behaviour during drought recovery. Plant, cell \& environment 43:1613-1624

57. Wang JW, Schwab R, Czech B, Mica E, Weigel D (2008) Dual effects of miR156-targeted SPL genes and CYP78A5/KLUH on plastochron length and organ size in Arabidopsis thaliana. The Plant cell 20:1231-1243

58. Wang Y, Liu W, Wang X, Yang R, Wu Z, Wang H, Wang L, Hu Z, Guo S, Zhang H, Lin J, Fu C (2020) MiR156 regulates anthocyanin biosynthesis through SPL targets and other microRNAs in poplar. 
Hortic Res 7:118

59. Wasternack C, Hause B (2013) Jasmonates: biosynthesis, perception, signal transduction and action in plant stress response, growth and development. An update to the 2007 review in Annals of Botany. Annals of botany 111:1021-1058

60. Wasternack C, Strnad M (2016) Jasmonate signaling in plant stress responses and development active and inactive compounds. New biotechnology 33:604-613

61. Wei T, Wang Y, Xie Z, Guo D, Chen C, Fan Q, Deng X, Liu JH (2019) Enhanced ROS scavenging and sugar accumulation contribute to drought tolerance of naturally occurring autotetraploids in Poncirus trifoliata. Plant biotechnology journal 17:1394-1407

62. Wei Y, Liu W, Hu W, Yan Y, Shi H (2020) The chaperone MeHSP90 recruits MeWRKY20 and MeCatalase1 to regulate drought stress resistance in cassava. The New phytologist 226:476-491

63. Wingler A, Roitsch T (2008) Metabolic regulation of leaf senescence: interactions of sugar signalling with biotic and abiotic stress responses. Plant biology 10 Suppl 1:50-62

64. Wu G, Park MY, Conway SR, Wang JW, Weigel D, Poethig RS (2009) The sequential action of miR156 and miR172 regulates developmental timing in Arabidopsis. Cell 138:750-759

65. Wu G, Poethig RS (2006) Temporal regulation of shoot development in Arabidopsis thaliana by miR156 and its target SPL3. Development 133:3539-3547

66. Xing Q, Liao J, Cao S, Li M, Lv T, Qi H (2020) CmLOX10 positively regulates drought tolerance through jasmonic acid -mediated stomatal closure in oriental melon (Cucumis melo var. makuwa Makino). Scientific reports 10:17452

67. Ye BB, Shang GD, Pan Y, Xu ZG, Zhou CM, Mao YB, Bao N, Sun L, Xu T, Wang JW (2020) AP2/ERF Transcription Factors Integrate Age and Wound Signals for Root Regeneration. The Plant cell 32:226241

68. Zhang P, Potrykus I, Puonti-Kaerlas J (2000) Efficient production of transgenic cassava using negative and positive selection. Transgenic research 9:405-415

\section{Figures}


A

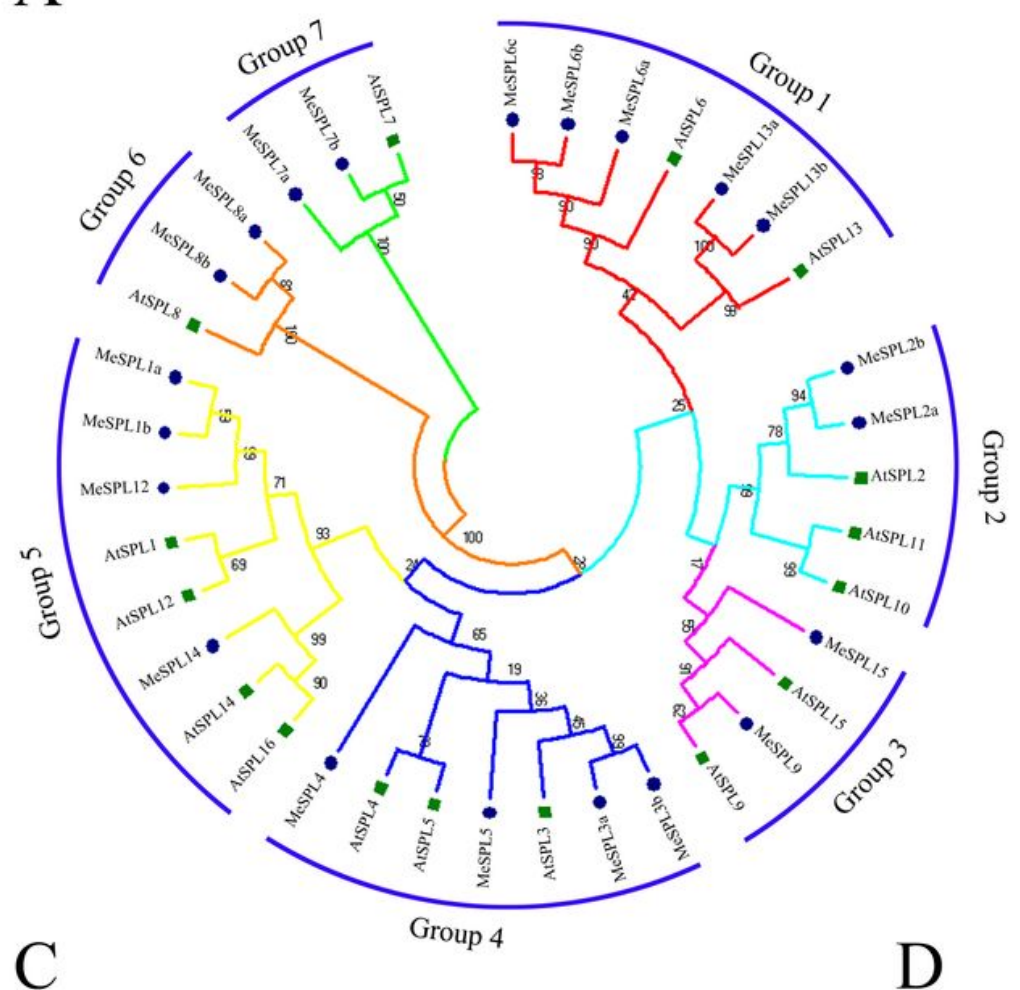

B
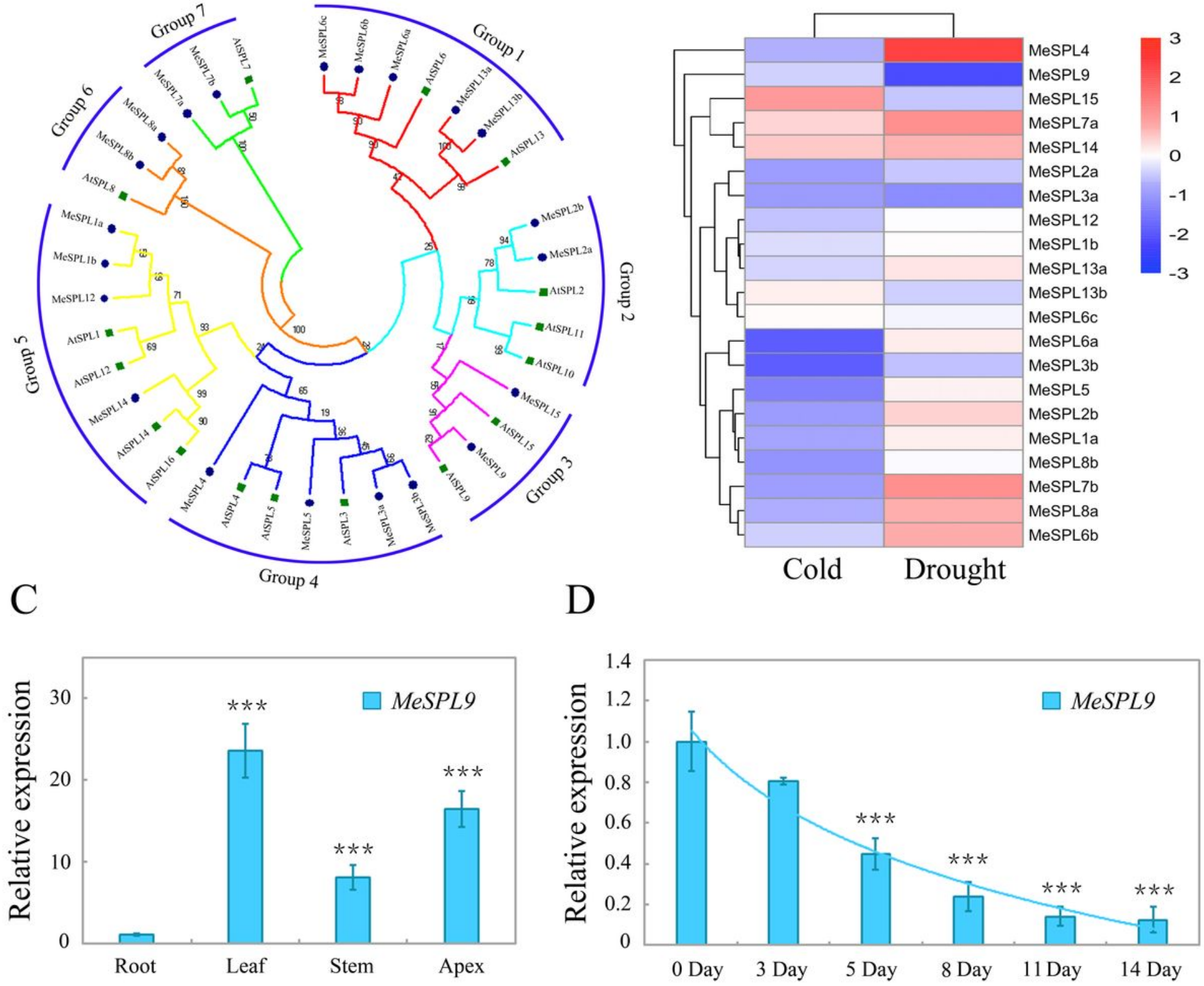

Figure 1

Phylogenetic and expression analyses of MeSPL transcription factors under abiotic stress conditions. (A) Phylogenetic analysis of cassava and Arabidopsis SPL genes. Numbers above or below tree branches show bootstrap values. The seven subgroups are indicated with different colors. (B) Expression profiles of MeSPLs under cold and drought conditions, respectively, as revealed by RNA-seq. (C) Spatial-temporal expression patterns of MeSPL9. (D) Expression of MeSPL9 under drought conditions. Drought treatment was conducted by water depletion for 0-14 days. Error bars indicate SDs obtained from three independent experiments. Significance of differences was determined by Student's t-test. $* \star \star, P<0.001$. 
A

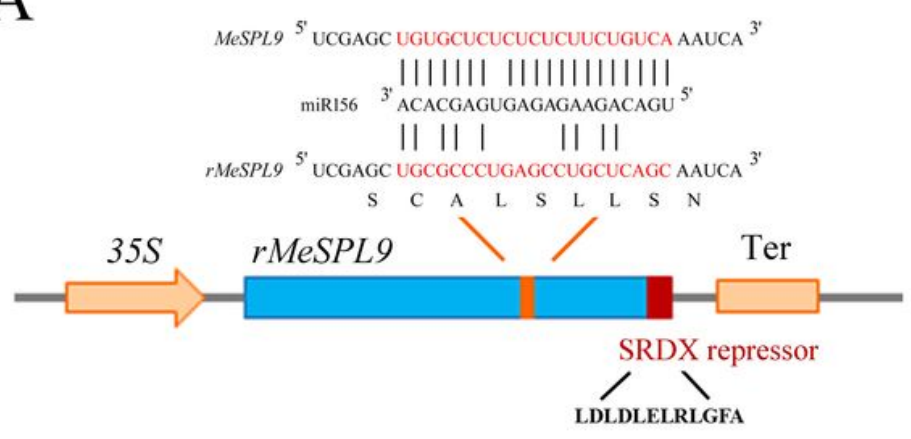

$\mathrm{C}$
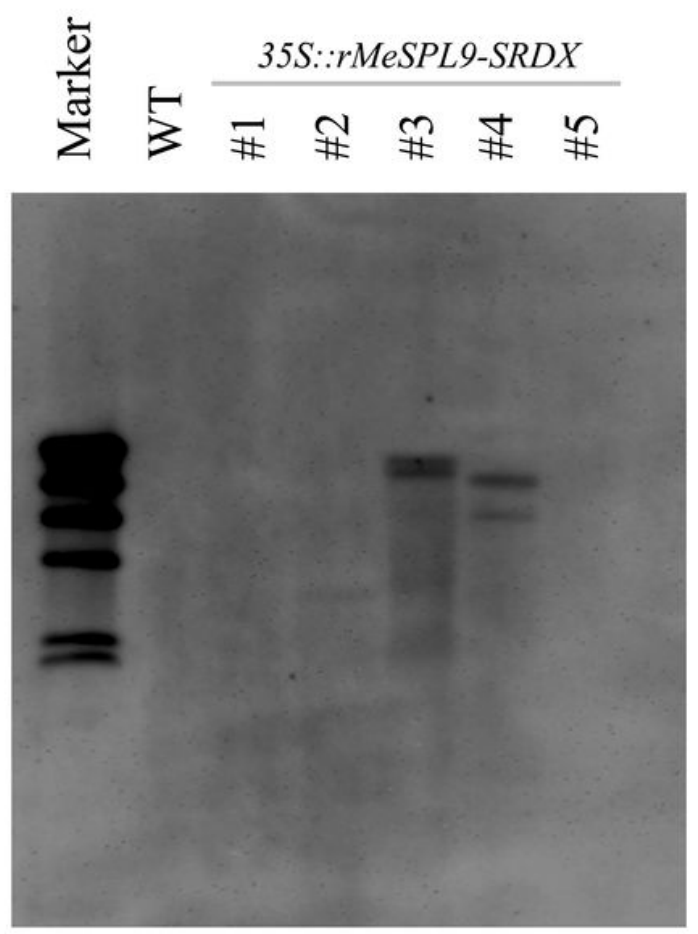

$\mathrm{D}$

$\mathrm{E}$
B
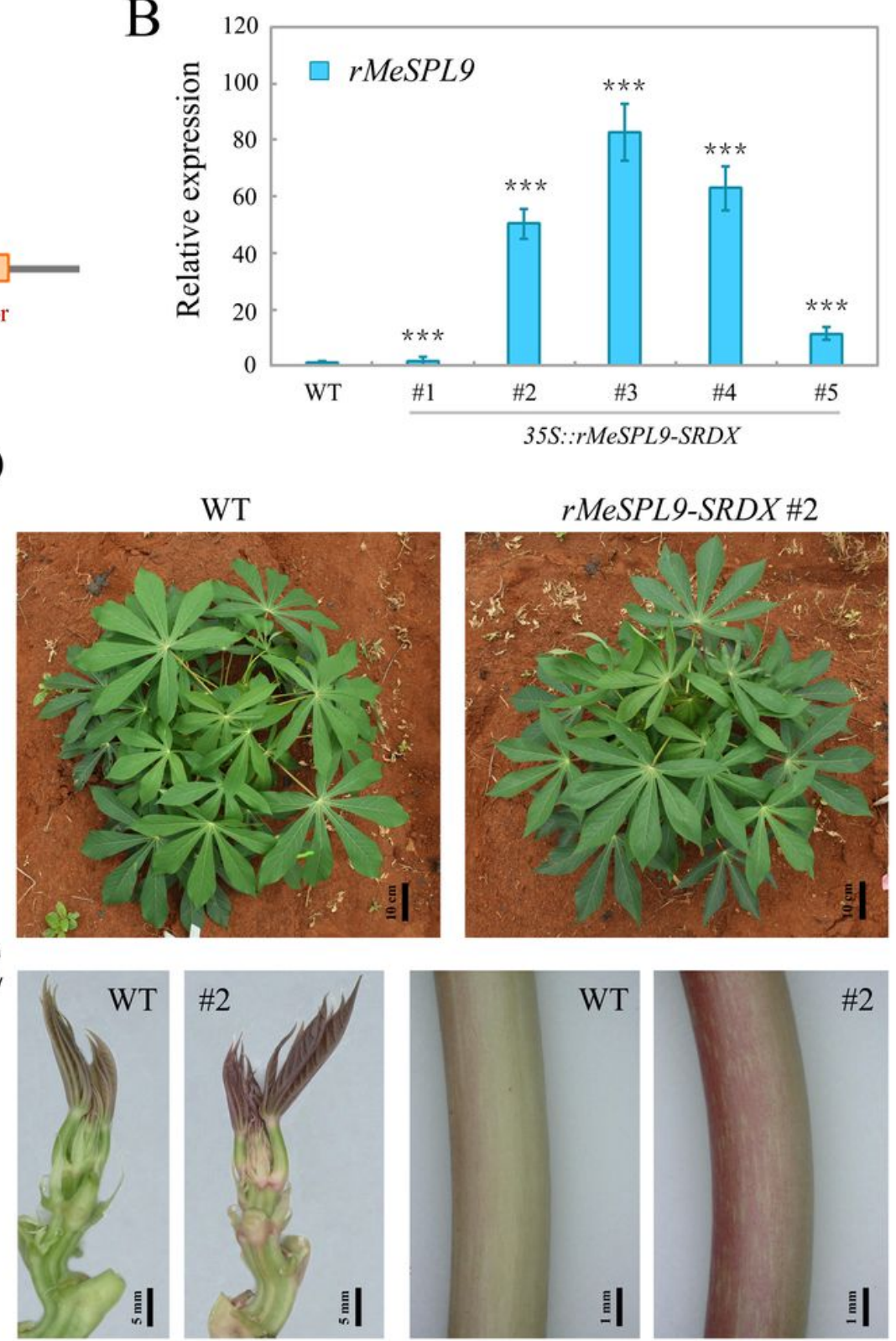

\section{Figure 2}

Molecular and phenotypic analysis of rMeSPL9-SRDX transgenic cassava plants. (A) Schematic presentation of the construction of p35S::rMeSPL9-SRDX and base mutation information of rMeSPL9 resistant to miR156 without changing protein sequence. 35S, CaMV 35S promoter; Ter, NOS terminator. (B) Relative expression of MeSPL9 in WT and transgenic lines. Error bars indicate SDs obtained from three independent experiments. Significance of differences was determined by Student's t-test. $\star \star \star, ~ P<$ 0.001. (C) Transgene integration validation in WT and transgenic lines by southern blotting. (D) Phenotype evaluation of WT and transgenic plants in field. (E) Shoot tips (left) and petiole (right) of WT and transgenic lines. 
A
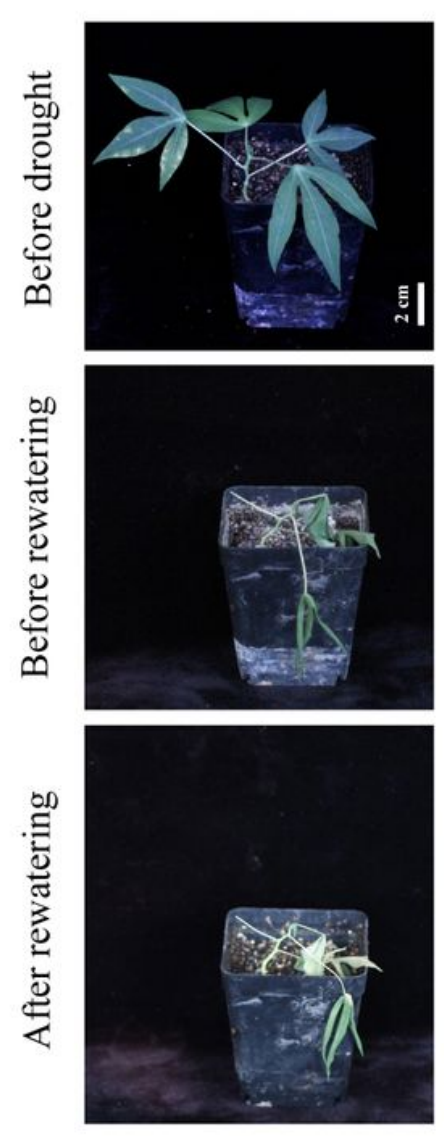

rMeSPL9-SRDX \#2
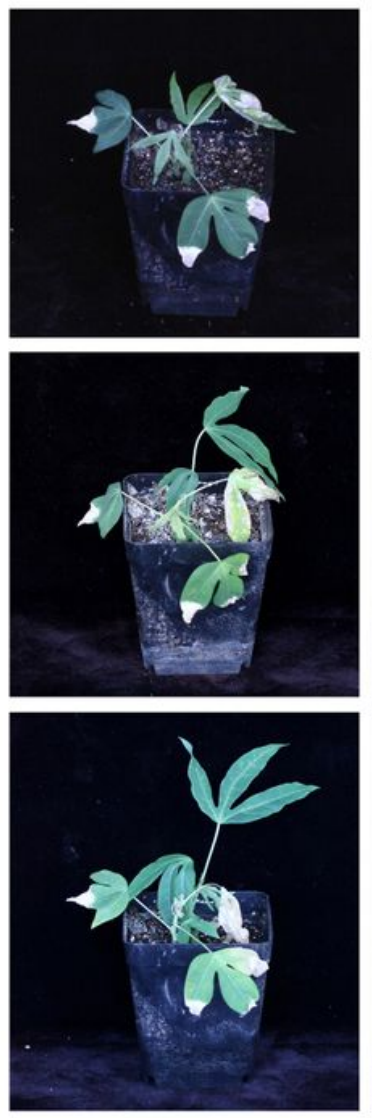

rMeSPL9-SRDX \#3
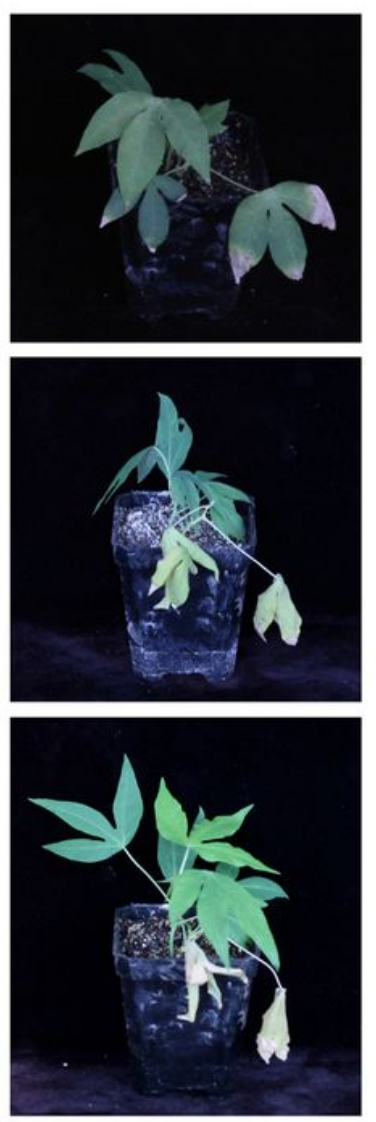

B
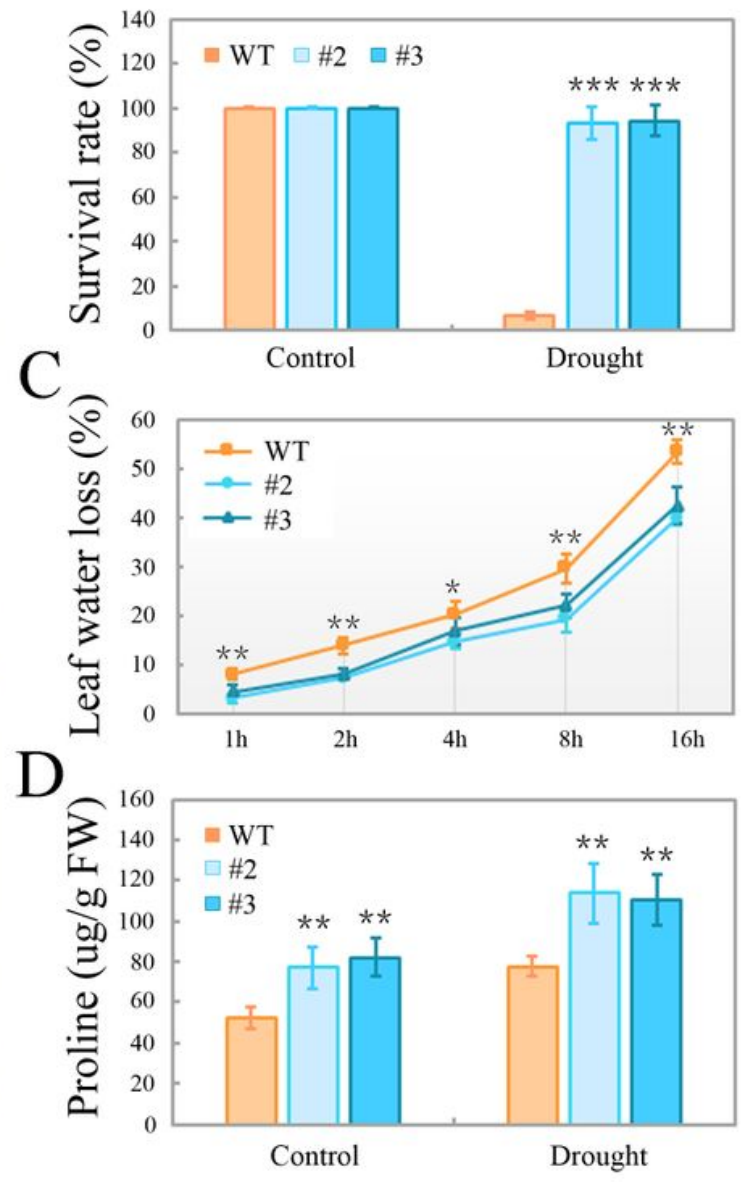

\section{Figure 3}

Down-regulation of MeSPL9 in cassava increases drought tolerance. (A) Representative phenotypes of WT and rMeSPL9-SRDX plants after drought treatment. Survival rate (B), leaf water loss rate (C), and proline content (D) in WT and rMeSPL9-SRDX lines under control and drought conditions. Error bars indicate SDs obtained from three independent experiments. Significance of differences was determined by Student's t-test. *, $\mathrm{P}<0.05 ; * \star, \mathrm{P}<0.01 ; * \star \star, \mathrm{P}<0.001$. 
A

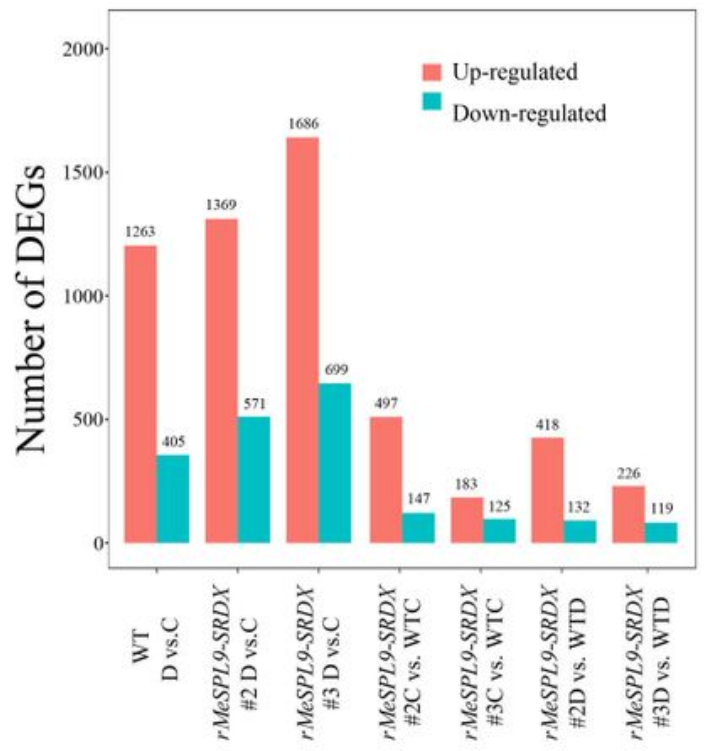

$\mathrm{B}$

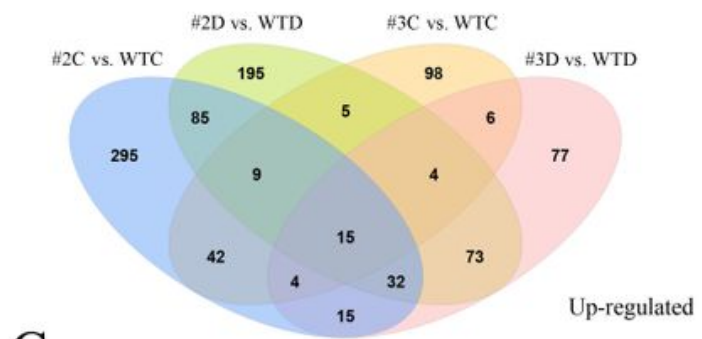

$\mathrm{C}$

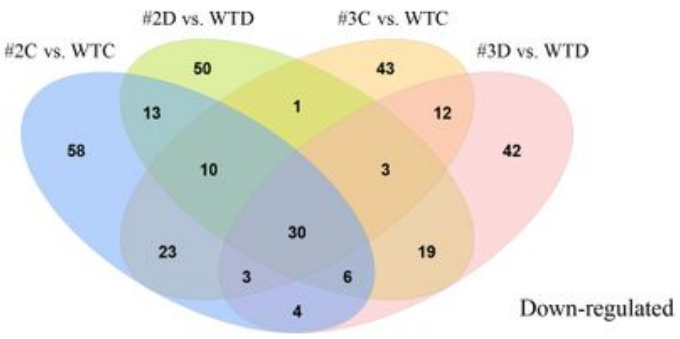

$\mathrm{E}$

Drought

GO terms Top 20

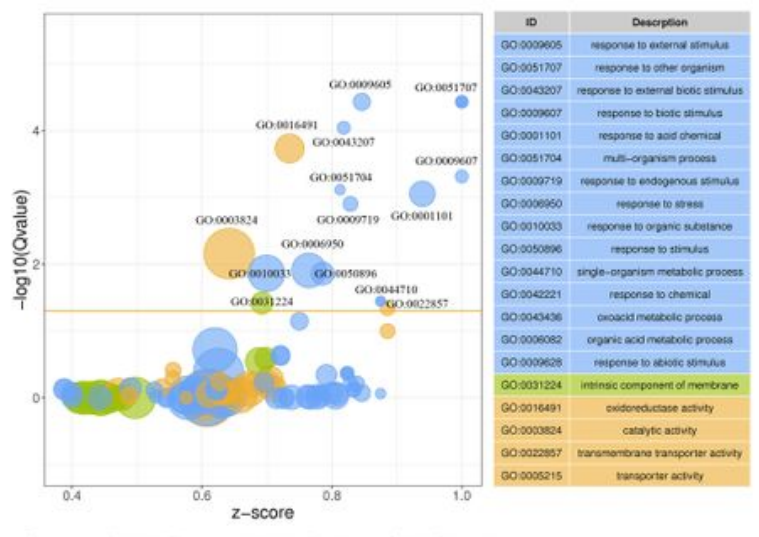

Categroy - Biological Procoss - Molbeular Function - Celluar Component

G
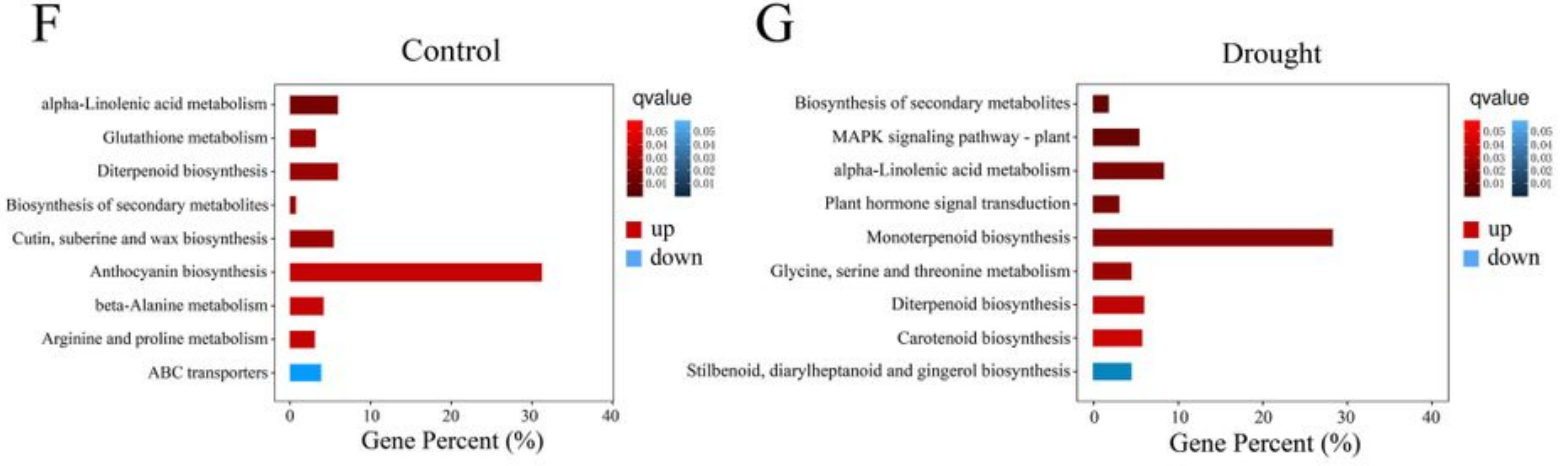

\section{Figure 4}

Identification of MeSPL9-regulated genes and related pathways. (A) Numbers of up- and down-regulated genes in WT and rMeSPL9-SRDX lines under control (C) and drought (D) conditions, as revealed by RNAseq. (B-C) Venn diagrams showing the common and specific up- (B) and down-regulated (C) genes in various pairwise comparisons. (D-E) Gene ontology enrichment analysis of MeSPL9-regulated genes under control (D) and drought (E) conditions, respectively. (F-G) KEGG pathway enrichment of MeSPL9- 
responsive genes under control $(F)$ and drought $(G)$ conditions, respectively. Up, pathways enriched based on up-regulated genes by MeSPL9. Down, pathways enriched based on down-regulated genes by MeSPL9.

A

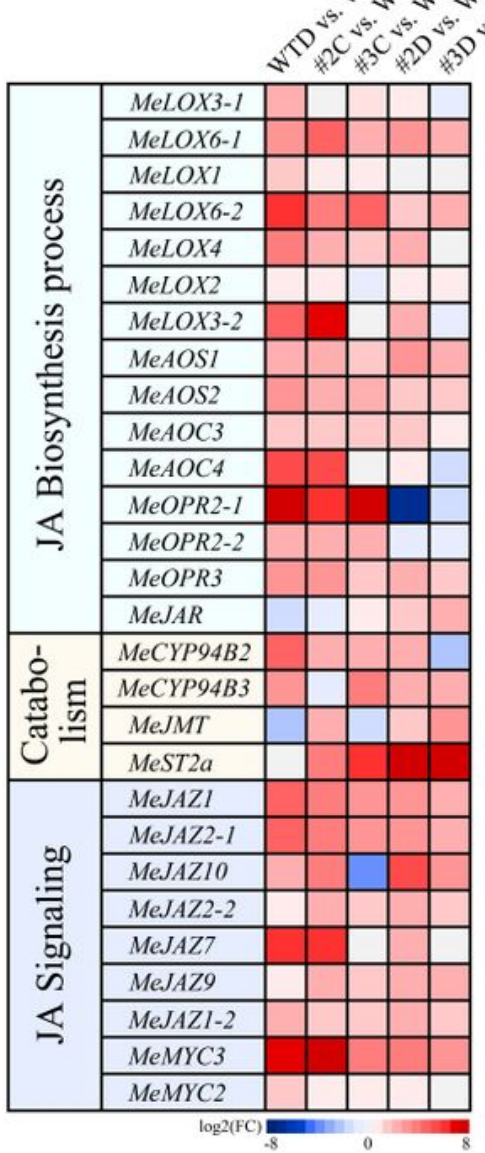

$\mathrm{D}$

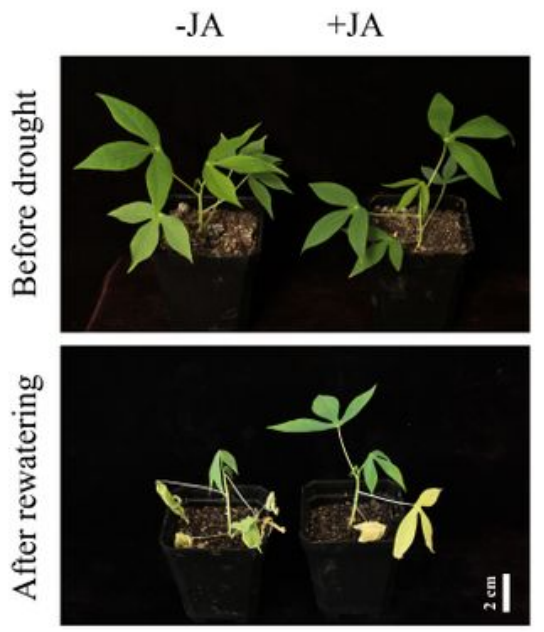

alpha-Linolenic acid

$\mathrm{B}$

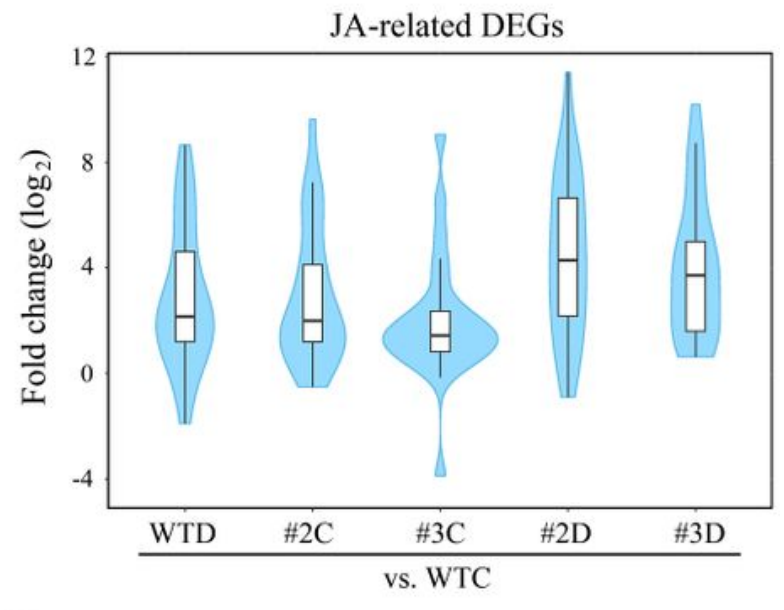

$\mathrm{C}$

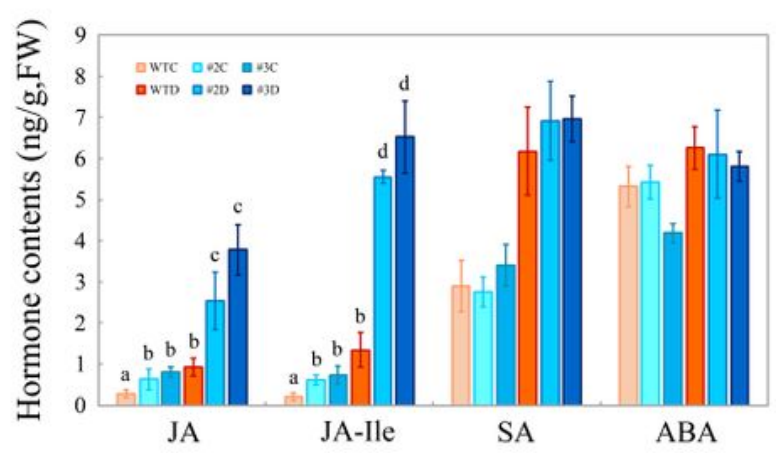

$\mathrm{F}$

$\mathrm{E}$ WT

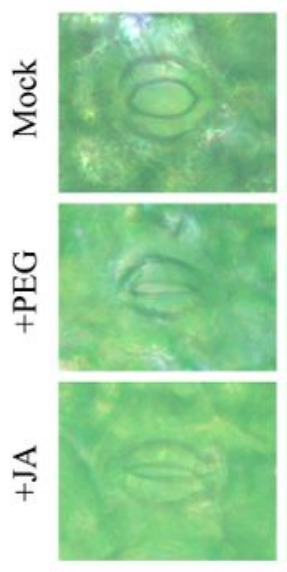

\#2
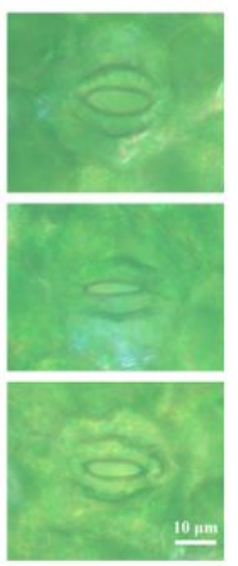

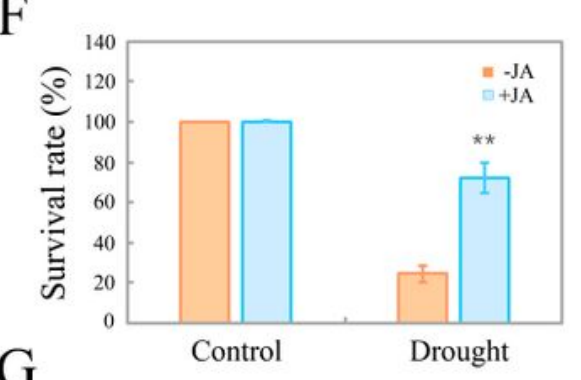

$\mathrm{G}$

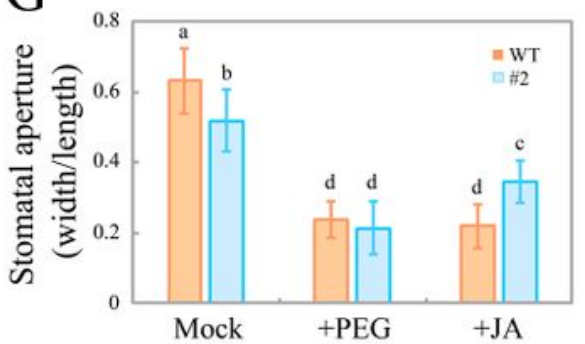

Figure 5

Enhanced accumulation of JA contributes to the drought-resistant phenotype of rMeSPL9-SRDX lines. (A) Expression profiles of genes participating in JA biosynthesis, catabolism, and signaling pathways in 
various pairwise comparisons. (B) Fold change of JA-related genes in WT and rMeSPL9-SRDX plants exposed to drought (D) and control (C) conditions. (C) Endogenous hormone levels in WT and rMeSPL9SRDX plants under drought (D) and control (C) conditions. JA: jasmonic acid, JA-lle: JA-isoleucine, SA: salicylic acid, ABA: abscisic acid. (D, F) Representative phenotypes (D) and survival rate (F) of wild-type cassava seedlings with or without application of exogenous JA after drought treatment. Bar $=2 \mathrm{~cm}$. Error bars indicate SDs obtained from three independent experiments. Significance was determined by Student's t-test. ${ }^{*}, P<0.01$. (E) Stomatal phenotype of WT and rMeSPL9-SRDX lines under PEG and JA treatments. Bar=10 $\mu \mathrm{m}$. (G) Stomatal size of WT and rMeSPL9-SRDX lines under PEG and JA treatments. Error bars indicate SDs obtained from three independent experiments. Different letters above bars indicate significant differences between WT and rMeSPL9-SRDX plants at $P<0.05$ (Duncan's range test). Significance of differences was determined by Student's t-test. **, $P<0.01$. 

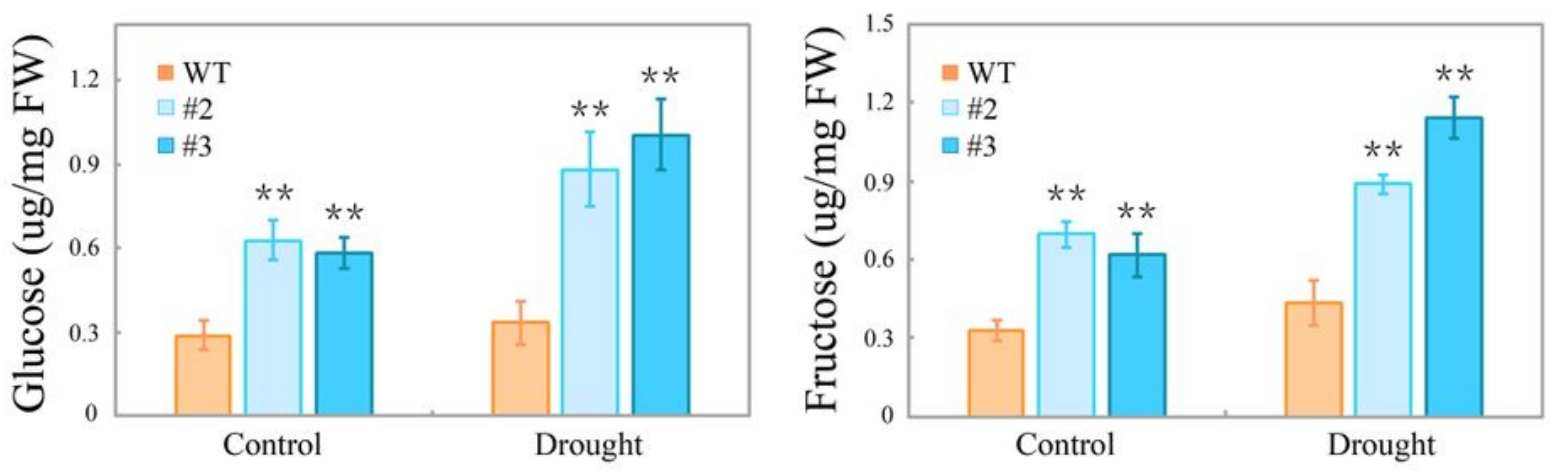

$\mathrm{C}$

$\mathrm{D}$
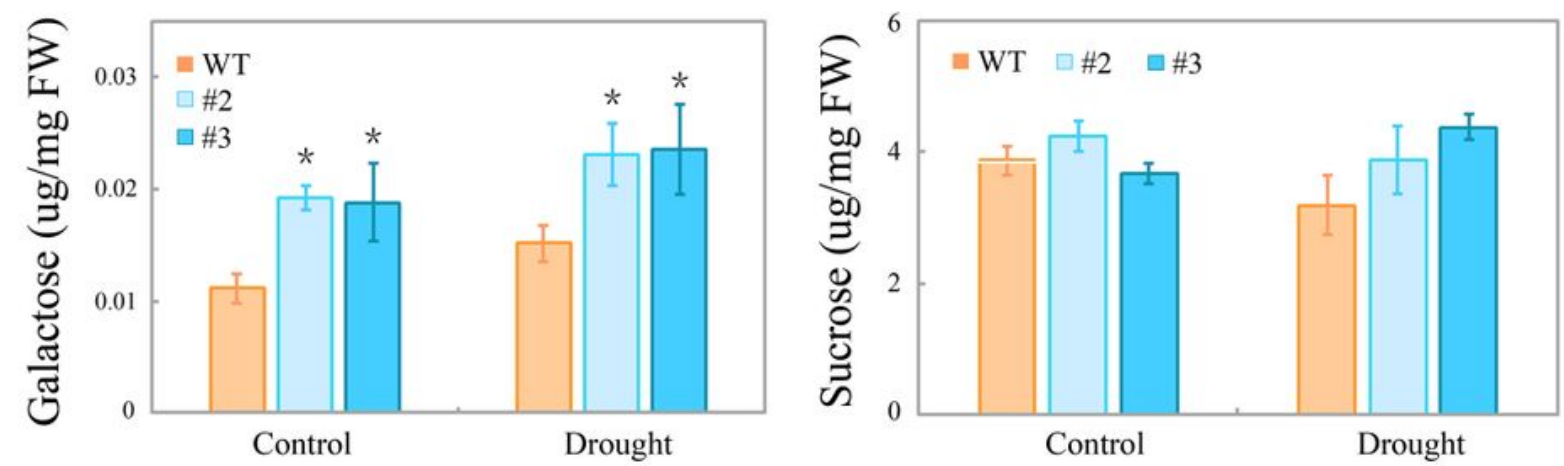

$\mathrm{E}$
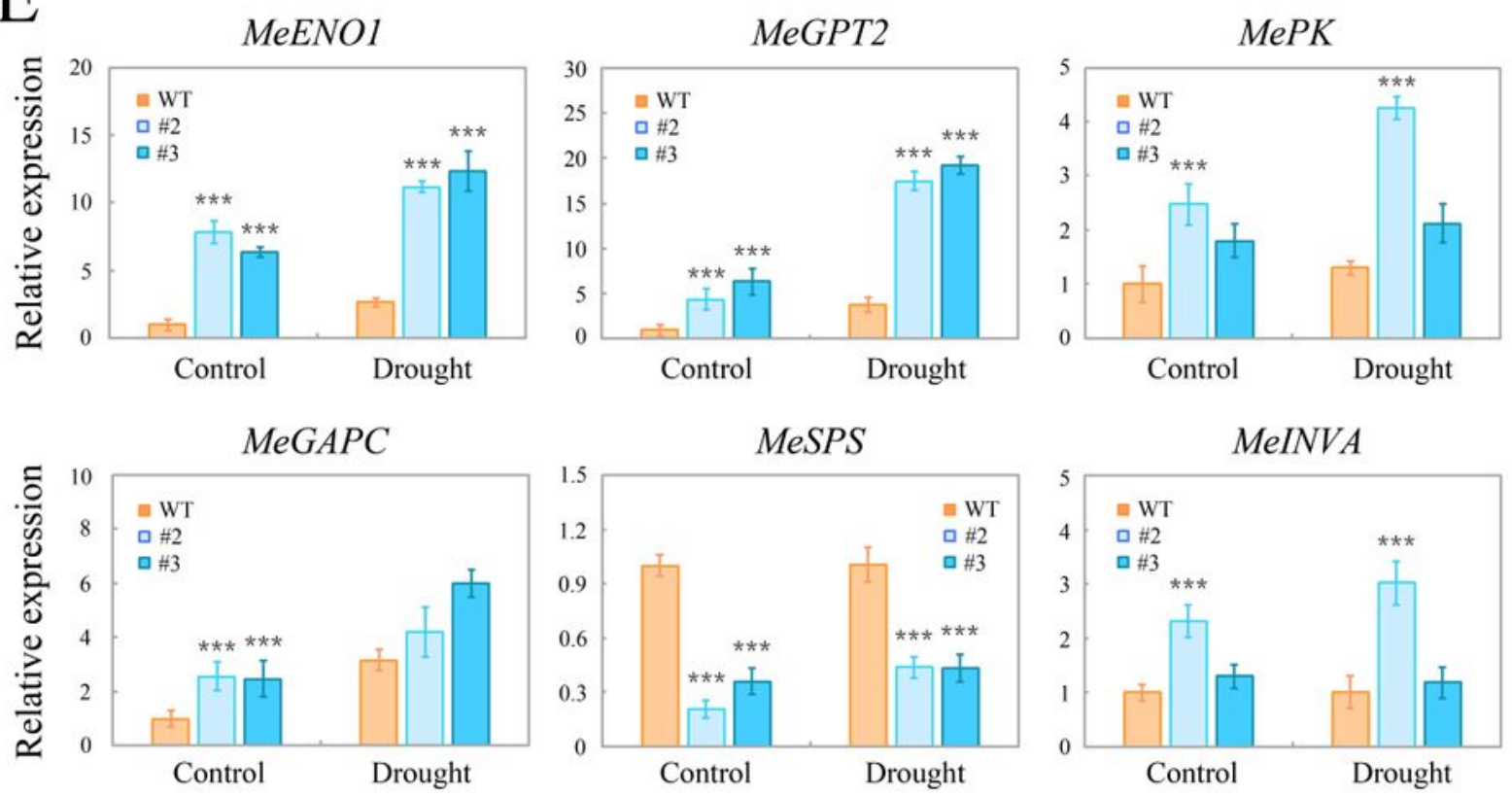

\section{Figure 6}

Expression of carbon metabolism-related genes and soluble sugar levels in WT and rMeSPL9-SRDX lines under drought conditions. (A-D) Contents of glucose (A), fructose (B), galactose (C), and sucrose (D) in WT and rMeSPL9-SRDX lines after drought treatment. (E) Relative expression levels of MeENO1, MeGPT2, MePK, MeGAPC, MeSPS, and MeINVA in WT and rMeSPL9-SRDX lines under control and 
drought conditions. Error bars indicate SDs obtained from three independent experiments. Significance of differences was determined by Student's t-test. *, $\mathrm{P}<0.05 ; * \star, \mathrm{P}<0.01 ; * \star \star, \mathrm{P}<0.001$.

\section{Supplementary Files}

This is a list of supplementary files associated with this preprint. Click to download.

- SupplementaryFigureandtables.pdf 